\title{
TORSION OF THE GALLBLADDER IN A KYPHO-SCOLIOTIC PATIENT
}

\author{
G. L. Mouzas, D.M. \\ Surgical Registrar, Bethnal Green Hospital, London, E.2
}

In surgical practice torsion of the gallbladder is an uncommon condition. In 1898 the first of such cases was reported by Wendel ${ }^{11}$ in a 23 -yearold girl who had been under observation for I6 months with a known wandering abdominal tumour. At operation she was found to have torsion of the gallbladder with a local abscess. Following this, other single-case reports appeared so that in 1945, when Leger, Debeyre and Mazingarbe $^{7}$ described three cases, they were able to survey a total of 9I cases from the literature. Although there are now 200 cases reported in the world literature, that of Kazmann and Guthorn $^{4}$ is the only other which has been associated with abscess formation as in the first case described by Wendel. The only case so far reported in a patient with kypho-scoliosis was that of Rais and Thulin ${ }^{9}$ in 1957 .

This report is of an elderly woman with kyphoscoliosis who presented with abdominal pain, and at laparotomy was found to have torsion of the gallbladder.

\section{Case Report}

An emaciated lady, 79 years of age, was admitted to hospital with a two-day history of abdominal pain and vomiting. On examination she had an obvious kypho-scoliosis, the abdomen was tender over the right hypochondrium and right iliac fossa, but no mass was felt. The chest showed diminished air entry at the right base and signs of generalized bronchitis. In the cardio-vascular system the heart was enlarged and the pulse fibrillating. B.P. I70/70. A straight X-ray of the abdomen revealed distended coils of gut without fluid levels and confirmed the kypho-scoliosis. A provisional diagnosis of intestinal obstruction was made.

At operation the abdomen was opened through a right paramedian incision. The abdominal cavity contained blood-stained fluid. A mass was palpated in the area of the liver, which was found to be the gallbladder twisted more than 180 degrees around its pedicle. Cholecystectomy was per- formed without difficulty (Fig. I). The gall- $\vec{\omega}$ bladder was distended with blood-stained fluido but no gallstones were found. The abdomen was 3 closed with drainage. Post-operatively the patienti was treated with antibiotics for her respiratory infection, and the auricular fibrillation was controlled with Digoxin. She made a satisfactory's recovery and was discharged home four weeks after admission. Microscopically the gallbladdero showed areas of infarction.

\section{Discussion}

Torsion of the gallbladder may be complete or incomplete. In the incomplete form the rotation of the gallbladder on its pedicle is less then I 80 degrees. With this type the onset may insidious and symptoms mild, while there may ồ. may not be a palpable mass. It is difficult make the correct diagnosis as there are no obvious clinical signs and it is not suspected until abscess formation occurs. In complete torsion the rotation exceeds 180 degrees, and with this the onset is $\stackrel{\square}{\square}$ more acute so that the patient may present with $\overrightarrow{\overrightarrow{0}}$ severe pain in the right hypochondrium simu- $\frac{0}{3}$ lating biliary colic. Other common symptoms are continuous pain with nausea, recurrent vomiting, flatulence and indigestion, usually without a previous history of cholecystitis or jaundice.

On examination, in the early stage, tenderness on the right side of the abdomen may be confused with other surgical conditions such as chole- 0 cystitis, appendicitis and disorders of the colon. 3 Symptoms and signs of peritonitis only develop윽 when the gallbladder becomes gangrenous and $>$ this then adds to the difficulty of diagnosis. Correct pre-operative diagnosis was made only in two cases reported by Krabbell ${ }^{6}$ and Kettner. ${ }^{5}$ or

The aetiology of torsion of the gallbladder is $N$ not known. Usually it occurs in elderly women between the ages of $60-80(92 \%)$. A few cases are known to have occurred in young children, as 0 reported by Cuervo, ${ }^{1}$ Dabadie $^{2}$ and Rawson.10

Reviewing the embryology and the different $\stackrel{\oplus}{+}$ anatomical arrangements of the gallbladder, $T$ 


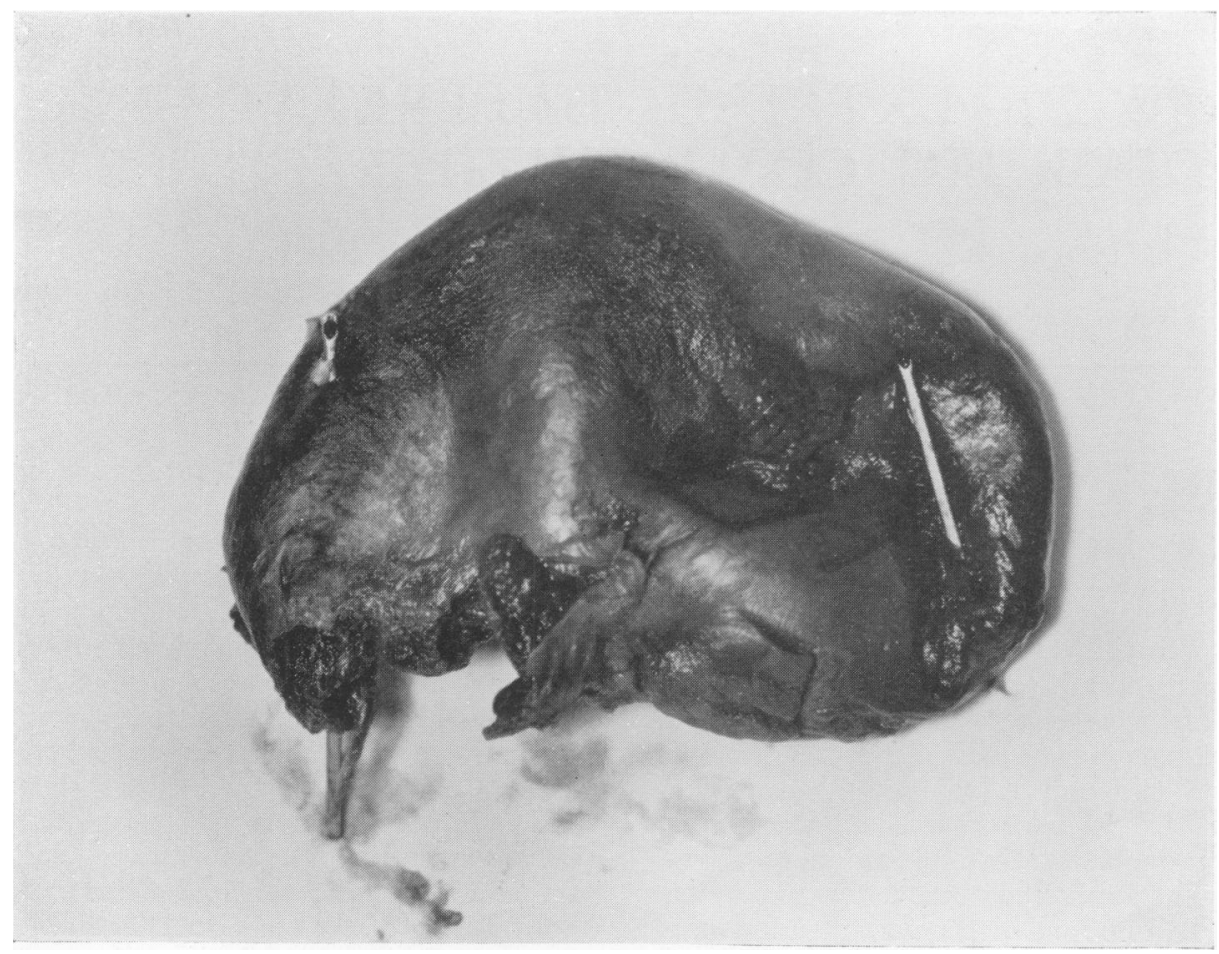

FIG. I.-Gangrenous gallbladder removed at operation.

Meeker and Lisemby $^{8}$ suggested that torsion develops when the gallbladder is completely surrounded by peritoneum and lies free in the abdominal cavity, except for its attachment along the course of the cystic duct and cystic artery. Gross $^{3}$ differentiates two types. In one the gallbladder has a mesentery, whilst in the other the cystic duct alone is supported by mesentery, allowing the gallbladder to hang freely. He suggested that the loss of fat from the surrounding parts and atrophy of the tissues in later age allows for greater ptosis of the viscera, thereby possibly predisposing to a floating gallbladder. Kypho-scoliosis is a condition in which the changed position of the abdominal organs may contribute to the development of visceroptosis, thus predisposing to torsion of the gallbladder. This has been suggested by Rais and Thulin. ${ }^{9}$

Torsion of the gallbladder could arise from active waves of peristalsis occurring in the fasting stomach, when the gallbladder would rotate in a clockwise direction. Alternatively, it may be caused by the movement of normal peristalsis in the transverse colon, which, being from right to left, would cause rotation in a counter-clockwise direction.

\section{Summary}

A case of torsion of the gallbladder in an elderly female patient with kypho-scoliosis is described. It is suggested that it is important to consider this as a differential diagnosis when such a patient presents with abdominal pain.

\section{Acknowledgments}

I wish to thank Sir John Nicholson, who allowed me to operate on his patient.

\section{REFERENCES}

I. CUERVO, S., and CUERVO, T. (1939), Arch. Soc. Estud. clin. Habana, 33, 295.

2. DABADIE, M. J. (1946), F. Med. Bordeaux, 123, 298.

3. GROSS, R. (1936), Arch. Surg. (Chicago), 32, 131.

4. KAZMANN, H. A., and GUTHOERN, P. J. (1953), Amer. $\mathcal{F}$. Surg., 85, 580.

5. KETTNER, G. (1930), Zbl. Chir., 57, 589.

6. KRABBEL, H. (1920), Dtsch. Z. Chir., 76, 154.

7. LEGER, L., DEBEYRE, J., and MAZINGARBE, A. (1945), F. Chir. (Paris), 6I, 21 .

8. MEEKER, W. R., and LISEMBY, J. O. (1939), Sth. med. F. (Bgham, Ala.), 25, 609.

9. RAIS, O., and THULIN, C. A. (1957), Acta chir. scand., II3, Fasc. 4, 289.

10. RAWSON, H. D. (1953), Aust. N.Z. F. Surgery, 22, 315.

I1. WENDEL, A. (1898), Ann. Surg., 27, 199. 